\title{
Über Carnaubon: Ein glycerinfreies Phosphatid, lecithinăhnlich konstituiert mit Galaktose als Kern.
}

\author{
Von
}

Edward K. Dunham, M. D., New York

und C. A. Jacobson, Ph. D., Reno, Nevada.

(Der Redaktion zugegangen am 5. Januar 1910.)

Vor zwei Jahren hat der eine von uns kurz über ein aus Rindernieren mit Alkohol extrahiertes Lipoid berichtet, das Carnaubinsäure liefert ${ }^{1}$ ). Damals wurde über die Eigenschaften dieses Lipoids nur sehr wenig mitgeteilt, da es noch Gegenstand der Untersuchung war. Wir möchten jetzt die Substanz eingehender beschreiben, und machen den Vorschlag, sie Carnaubon zu nennen; einen kurzen Namen, der trotzdem die komplizierte Zusammensetzung der Substanz andeuten würde, zu finden, ist uns nicht gelungen.

In der früheren Mitteilung ist die Methode, die zur Darstellung des Carnaubons angewandt wurde, nur skizziert; sie soll zunächst ausführlich beschrieben werden.

I. Die Gewinnung des Carnaubons. Auf dem Markt gekaufte Rindernieren wurden möglichst vom sichtbaren Fett befreit und mit der Wurstmaschine zerkleinert. Etwa $35 \mathrm{~kg}$ des Breies wurden mit $10195 \%$ igem Alkohol gekocht. Der braune, fluorescierende Extrakt wurde durch ein Tuch vom Gewebe getrennt und weggeworfen. Hierauf extrahierte man den Rückstand erst einmal mit $95 \%$ igem und dann mehrmals mit 85\% igem Alkohol. Die Auszüge wurden heiß filtriert, vereinigt und in Eiswasser bis auf etwa Null Grad abgekühlt. Nach 12-14 Stunden setzte sich ein hellgelber wachsähnlicher Niederschlag zu Boden. Er wurde auf einem Filter gesammelt, trocken gesaugt, dann in $500 \mathrm{ccm}$ Benzol, in dem er fast vollständig löslich ist, aufgenommen, und die Lösung filtriert und durch Destillation unter vermindertem Druck bis zum dicken Sirup

1) Dunham, E. K., The Isolation of Carnaubic Acid from Beef Kidney: - The Journal of Biological Chemistry, Bd. IV, S. 297. 
eingeengt. Letzterer wurde mit $500 \mathrm{ccm}$ Äther im Scheidetrichter gut geschüttelt und das Gemisch ruhig stehen gelassen. Nach 6 bis 7 Stunden schied sich ein feiner, weißer Niederschlag ab. Der Äther wurde dann abgehoben, und der Niederschlag so lange mit frischem Äther behandelt, bis dieser sich nicht mehr färbte. Die weitere Reinigung des Niederschlags geschah durch viermaliges Umkrystallisieren aus heißem $95 \%$ oigem Alkohol. Zum Schluß wurde die Substanz noch im SoxhletApparat mit Äther extrahiert. Das auf diese Weise gewonnene Produkt ist es, dessen wir uns zu den meisten unserer Untersuchungen bedienten. Es ist nicht ausgeschlossen, daß er ein Gemenge angewandter Substanzen darstellt, die von einander im Stickstoffgehalte abweichen. Ja, wir glauben sogar, daß dies der Fall ist, denn wir haben in der letzten Zeit beobachtet, daß Amylalkohol eine weitere Trennung ermöglicht. Wir kommen auf diesen Gegenstand noch zurück. Für die Hauptzwecke unserer Untersuchungen ist indessen das nach der angegebenen Methode gereinigte Carnaubon vollkommen brauchbar.

Wir erhielten ungefähr $6 \mathrm{~g}$ Carnaubon aus $35 \mathrm{~kg}$ feuchten Nierengewebes. Die Ausbeute wird durch Fäulnis nicht merklich beeinflußt. Es ist deswegen wahrscheinlich kein Nachteil, daß wir die Nieren nicht direkt vom Schlachthaus bezogen, sondern auf dem Markt kauften.

II. Hypothetische Formel für Carnaubon. Ehe wir an die ausführliche Beschreibung unserer Untersuchungen herangehen, möchten wir die erhaltenen Resultate in einer hypothetischen Formel zum Ausdruck bringen. Diese Formel gründet sich auf die Annahme, daß das Carnaubon eine Amino-Galaktose ist, mit welcher drei Fettsäuren und Phosphorsäure in Verbindung stehen, die Phosphorsäure überdies mit zwei Cholingruppen. Die Fettsäuren sind Carnaubin-, Stearin- und Palmitinsäure. Die Formel lautet:

$\mathrm{C}_{6} \mathrm{H}_{9} \mathrm{NO} . \mathrm{C}_{24} \mathrm{H}_{47} \mathrm{O}_{2} \cdot \mathrm{C}_{18} \mathrm{H}_{35} \mathrm{O}_{2} \cdot \mathrm{C}_{16} \mathrm{H}_{31} \mathrm{O}_{2} . \mathrm{PO}_{4}\left(\mathrm{C}_{5} \mathrm{H}_{14} \mathrm{NO}\right)_{2}$

Empirisch zusammengezogen nimmt sie die Form $\mathrm{C}_{74} \mathrm{H}_{150} \mathrm{~N}_{3}$ $\mathrm{PO}_{13}$ an; es handelt sich also um ein Triazomonophosphatid.

Aus der Formel berechnet sich folgendes quantitative Verhältnis der Komponenten: 


$$
\begin{aligned}
& \text { C } 67,24 \text { Carnaubinsäure } \quad 27,90 \% \text { Cholin } 18,35 \% \\
& \text { H } 11,47 \text { Stearinsäure } 21,23 \% \text { Methyl } 6,83 \% \\
& \text { N } 3,19 \quad \text { Palmitinsäure } \quad 19,41 \% \text { Galaktose } 13,64 \% \\
& \mathrm{P} \quad 2,35 \text { Sämtliche Fettsäuren } \overline{68,54 \% \text {. }} \\
& \text { O } \frac{15,75}{100,00}
\end{aligned}
$$

Die experimentelle Verwirklichung der Erwartungen, zu denen man durch die Formel gelangt, erscheint von vornherein für einige der Komponenten ziemlich schwierig, für andere derselben verhältnismäßig leicht. Wir werden unsere Resultate so zusammenstellen, daß sich klar ergibt, bis zu welchem Grade die analystischen Werte mit den auf die Formel basierten theoretischen Erwartungen im Einklang sind.

III. Elementaranalysen des Carnaubons. Die Verbrennung nach der Li ebig schen Methode liefert nach unseren Erfahrungen beim Carnaubon zuverlässigere und etwas höhere Werte als das Dennstedtsche Verfahren. Die nach Liebigs Methode erhaltenen Resultate sind folgende:

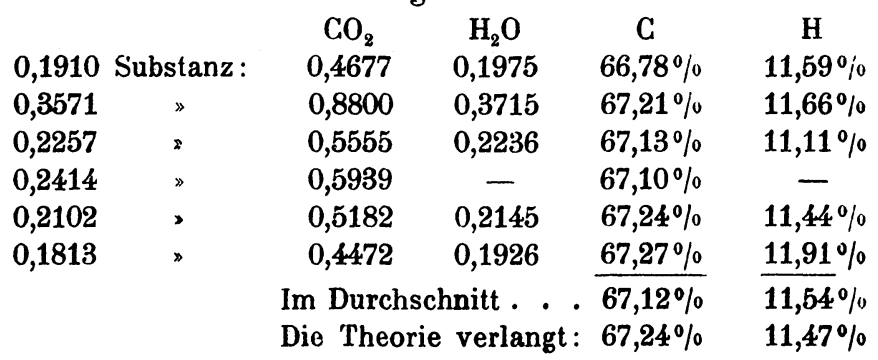

Der Phosphor wurde als Magnesiumpyrophosphat bestimmt,

\begin{tabular}{|c|c|c|c|c|}
\hline 0,4840 & Substanz: & 0,0358 & $\mathrm{Mg}_{2} \mathrm{P}_{2} \mathrm{O}_{7}$ & $2,06 \% \mathrm{P}$ \\
\hline 0,4200 & $\gg$ & 0,0359 & 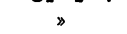 & $2,38 \%$ \\
\hline 0,4387 & » & 0,0338 & $»$ & $2,15 \% \times$ \\
\hline 0,6718 & » & 0,0517 & » & $2,14 \%$ \\
\hline 0,5951 & , & 0,0481 & ๖ & $2,25 \%$ \\
\hline 0,3663 & $»$ & 0,0296 & * & $2,25 \%$ \\
\hline 0,6751 & 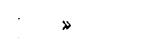 & 0,0489 & * & $2,02 \%$ \\
\hline 0,6042 & , & 0,0473 & , & $2,18 \%$ \\
\hline
\end{tabular}
nachdem das Carnaubon durch eine Mischung gleicher Teile konzentrierter Schwefel- und Salpetersäure feucht verbrannt und aus der verdünnten Lösung durch salpetersaures Ammoniak und Ammonmolybdat die Phosphorsäure niedergeschlagen war. 
Den Stickstoff haben wir nach Kjeldahl bestimmt, unter Zusatz von schwefelsaurem Kalium beim Erhitzen mit der konzentrierten Schwefelsäure:

\begin{tabular}{|c|c|c|c|c|}
\hline \multicolumn{2}{|c|}{0,4480 Substanz: } & \multicolumn{2}{|c|}{$8,15 \mathrm{ccm} n / 10-$ Säure } & \multirow{2}{*}{$\begin{array}{l}2,55 \% \mathrm{~N} \\
2,75 \%\end{array}$} \\
\hline 0,4189 & , & 8,20 & $»$ & \\
\hline 0,4252 & $»$ & 8,10 & » & $2,67 \%$ \\
\hline 0,4261 & ע & 9,42 & 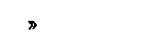 & $3,10 \%$ \\
\hline $\left.0,4016^{1}\right)$ & " & 8,22 & * & $2,87 \% \times 4$ \\
\hline $\left.0,4769^{1}\right)$ & , & 9,70 & • & $\left.2,86 \%{ }^{1}\right)$ \\
\hline $\left.0,4544^{1}\right)$ & 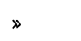 & 9,35 & D & $2,88 \%, 1)$ \\
\hline 0,4845 & » & 9,70 & " & $2,81 \%$ \\
\hline $\left.0,6196^{2}\right)$ & 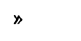 & 12,93 & > & $\left.2,93 \%{ }^{2}\right)$ \\
\hline $\left.1,4340^{2}\right)$ & $\Rightarrow$ & 29,88 & $"$ & $\left.2,93 \% »^{2}\right)$ \\
\hline
\end{tabular}

Andere Elemente haben wir nicht entdecken können. In manchen Präparaten fand sich zwar eine Spur Schwefel, wir betrachten denselben aber als Verunreinigung.

Die vorliegenden Resultate der Elementaranalyse stimmen nicht sehr genau mit der Theorie. Die vorgeschlagene Formel ist indessen nicht bloß auf die Resultate basiert, sondern auch auf die Bestimmungen der Fettsäuren, des Cholins und der Methylgruppen, deren Ergebnisse unten folgen. Die Elementaranalysen lassen vermuten, daß die benutzten Präparate des Carnaubons nicht ganz rein waren, denn das Verhältnis von Phosphor zu Stickstoff war nicht befriedigend einfach, sondern meistens ungefähr $=3: 7$. Solch ein Verhältnis würde auf ein sehr großes Molekül deuten. Es ist jedoch viel wahrscheinlicher, daß die Präparate des Carnaubons öfters etwas Stickstoff verloren hatten. Diese Vermutung hat in letzter Zeit insofern eine Stütze erhalten, als wir gefunden haben, daß es möglich ist, durch Amylalkohol ein Präparat des Carnaubons zu gewinnen, das diese teilweise Zersetzung nicht erlitten hat. Die Analyse eines solchen Präparates ergab:

$$
\begin{aligned}
& \text { 0,1385 Substanz 0,3387 } \mathrm{CO}_{2} \text { und 0,1406 } \mathrm{H}_{2} \mathrm{O} \text {. } \\
& 0,52687 \text { » } 0,01681 \mathrm{~N} \text {. } \\
& 0,51713 \text { * } 0,0443 \quad \mathrm{Mg}_{2} \mathrm{P}_{2} \mathrm{O}_{7} \text {. }
\end{aligned}
$$

1) Drei Bestimmungen bei demselben Präparat des Carnaubons.

2) Doppelbestimmung bei demselben Präparat des Carnaubons. 


\begin{tabular}{|c|c|c|c|c|}
\hline & G & $\mathbf{H}$ & $\mathbf{N}$ & $\mathbf{P}$ \\
\hline anden: & 66,70 & 11,38 & 3,19 & 2,38 . \\
\hline erechnet für $\mathrm{C}_{74} \mathrm{H}_{160} \mathrm{~N}_{3} \mathrm{PO}_{13}$ : & 67,24, & 11,47 & 3,19 & \\
\hline
\end{tabular}

Die Verbrennung wurde nach der Dennstedtschen Methode ausgeführt, die nach unserer Erfahrung, wie schon hervorgehoben, beim Carnaubon zu niedrige Resultate gibt.

IV. Die Fettsäuren aus Carnaubon. Eine einfache Hydrolyse des Carnaubons durch Säuren bezw. Alkalien ist nicht leicht $\mathrm{zu}$ bewirken. Diese Tatsache erklärt sich wahrscheinlich durch eine besonders feste Bindung zwischen Zucker und Fettsäuren.

Ist aber eine oxydierende Wirkung mit beteiligt, dann werden die Fettsäuren verhältnismäßig leicht abgespalten. Wir erhielten die besten Resultate bei Einwirkung mäßigverdünnter Salpetersäure. $5 \mathrm{~g}$ Carnaubon wurden mit $100 \mathrm{ccm}$ Salpetersäure, spezifisches Gewicht 1,15, in einem Kolben mit Rückflußkühler auf dem Wasserbad erst gelinde, dann stark erhitzt, im ganzen 24-30 Stunden lang. Hierbei werden salpetrigsaure Dämpfe abgegeben, und zuletzt schwimmt eine klare Schicht Fettsäuren auf der Oberfläche der Flüssigkeit. Diese Schicht erstarrt bei Zimmertemperatur zu einem hellgelben, harten Kuchen, der in Äther vollkommen löslich ist. Die so erhaltenen Fettsäuren wurden in Äther gelöst und im Scheidetrichter mit Wasser gewaschen, und enthielten dann nur Spuren Stickstoff. Nach drei Gewichtsbestimmungen an drei verschiedenen Carnaubonpräparaten wurden bezw. $68,14 \%, 69,50 \%$ und $68,29 \%$ Fettsäuren erhalten, im Durchschnitt 68,64\%. Die Theorie verlangt $68,54 \%$. Dies genau der Theorie entsprechende Resultat kann Zufall sein, indem wahrscheinlich etwas Fettsäure verloren ging, während kleine Spuren Verunreinigungen vorhanden gewesen sein mögen. Aber diese einander kompensierenden Zahlen sind sicherlich gering gewesen.

Carnaubinsäure läßt sich aus dieser Mischung der Fettsäuren trennen, entweder durch Esterbildung mit 5\% gesättiger alkoholischer Salzsäure, welche die Carnaubinsäure sehr leicht in ihren Äthylester verwandelt, oder durch Fällung als Lithiumseife, da carnaubinsaures Lithium in kochendem 50\% igem 
Alkohol unlöslich ist. Nach dieser Fällungsmethode haben wir 27,30\% Carnaubinsäure im Carnaubon gefunden. Die Theorie würde $27,90 \%$ erfordern. Nach einmaligem Umkrystallisieren aus Aceton war der Schmelzpunkt der Substanz 72-72,5 (unkorrigiert).

Die Analyse des Silbersalzes der so erhaltenen Carnaubinsäure ergab:

0,2174 Substanz $0,4835 \mathrm{CO}_{2}, 0,1893 \mathrm{H}_{2} \mathrm{O}, 0,0500 \mathrm{Ag}$.

Gefunden:

$$
\begin{array}{ccc}
\mathrm{C} & \mathrm{H} & \mathbf{A g} \\
60,67, & 9,77, & 23,02 .
\end{array}
$$

Berechnet für $\mathrm{AgC}_{84} \mathrm{H}_{47} \mathrm{O}_{2}: 60,60,9,97,22,70$.

Der Methylester, durch Erhitzen einer Lösung der Fettsäure in Methylalkohol mit Schwefelsäure erhalten, hatte den Schmelzpunkt $54,3^{\circ}$ (unk.), nachdem er aus Aceton einmal umkrystallisiert worden war. Die Verbrennung ergab:

$$
\begin{array}{lll}
0,1990 \text { Substanz } 0,5718 \mathrm{CO}_{2}, 0,2309 \mathrm{H}_{2} \mathrm{O} . \\
\mathrm{C} & \mathrm{H} \\
\text { Gefunden: } & 78,37, & 12,99 . \\
\text { Berechnet für } \mathrm{CH}_{3} \cdot \mathrm{C}_{24} \mathrm{H}_{47} \mathrm{O}_{2}: & 78,45, & 13,18 .
\end{array}
$$

Die übrigen Fettsäuren lassen sich weniger leicht trennen bezw. bestimmen.

Das heiße Filtrat vom carnaubinsauren Lithium wurde auf Zimmertemperatur abgekühlt. Es bildete sich ein weißer Niederschlag. Dieser wurde auf einem Filter gesammelt, mit $50 \%$ igem Alkohol gewaschen und durch verdünnte Salzsäure zerlegt. Die freien Fettsäuren wurden in ätherischer Lösung mit Wasser gewaschen, der Äther verjagt und der Rückstand aus 80\% igem Alkohol krystallisiert. Der Schmelzpunkt der auf diese Weise erhaltenen Säuren war nicht scharf, sondern schwankte zwischen 55 und $58^{\circ}$ (unk.).

Die Verbrennungen zweier Präparate aus zwei verschiedenen Carnaubonproben ergaben:

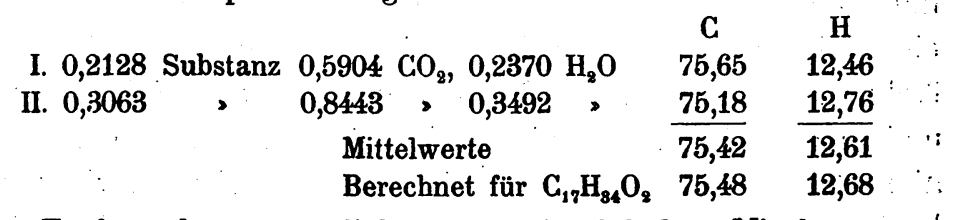

Es lag also vermutlich eine äquimolekulare Mischung von 
Stearin- und Palmitinsäure vor, die einen Schmelzpunkt von etwa $57,4^{\circ}$ zeigen sollte.

Wir haben kleine Mengen Stearin- und Palmitinsäure aus dieser Mischung isolieren können, indem wir die Lithiumseifen in absolutem Alkohol durch Kochen auflösten und die Lösung auf Zimmertemperatur abkühlten. Der Niederschlag, der sich hierbei bildete, wurde auf einem Filter gesammelt und mit Salzsäure zerlegt, die freie Säure gewaschen und zweimal aus Aceton umkrystallisiert. Eine Verbrennung lieferte folgende Resultate :

$$
\begin{array}{lrr}
0,1786 \text { Substanz } & 0,4983 \mathrm{CO}_{2}, 0,2016 \mathrm{H}_{2} \mathrm{O} . \\
\mathrm{C} & \mathrm{H} \\
\text { Gefunden: } & 76,08, & 12,63 . \\
\text { Berechnet für } \mathrm{C}_{18} \mathrm{H}_{30} \mathrm{O}_{2}: & 75,98, & 12,76 .
\end{array}
$$

Das Filtrat vom stearinsauren Lithium wurde zur Trockene eingedampft, mit verdünnter Salzsäure zerlegt und die freie Fettsäure in ätherischer Lösung mit Wasser gewaschen. Nach Abdampfen des Äthers wurde die Säure durch Kali in $80 \%$ igem Alkohol verseift und die Seifenlösung mit Baryumacetat fraktioniert gefällt. Der erste Niederschlag wurde vernachlässigt und aus dem Rest die Fettsäure durch verdünnte Salzsäure freigemacht und zweimal aus 80\%igem Alkohol umkrystallisiert. Obgleich die Ausbeute recht gering war, machten wir eine Verbrennung mit folgendem Erfolg:

$$
\begin{array}{lcc}
0,0928 \text { Substanz } & 0,2552 \quad \mathrm{CO}_{2}, 0,1047 \mathrm{H}_{8} \mathrm{O} . \\
& \mathrm{C} & \mathrm{H} \\
\text { Gefunden: } & 75,00, & 12,63 . \\
\text { Berechnet für } \mathrm{C}_{16} \mathrm{H}_{88} \mathrm{O}_{2}: & 74,92, & 12,59 .
\end{array}
$$

Alle diese Resultate sind unter einander und mit der Theorie in Einklang.

Man kann auch durch alkalische Kaliumpermanganatlösung die Fettsäuren aus dem Carnaubon erhalten; die Ausbeute ist dann aber nicht quantitativ, denn die Fettsäuren erleiden dabei eine teilweise Oxydation bezw. Spaltung, unter Bildung einfacherer Substanzen. Sie können sogar ganz vernichtet werden und man erhält dann gar keine der höheren Fettsäuren. Wenn aber die Wirkung nicht so weit getrieben wird, gewinnt man leicht etwa zwei Drittel der Fettsäuren. Wir haben diese 
Mischung der Fettsäuren nicht analysiert, dagegen einmal für sie den Mittelwert der Molekulargewichte bestimmt durch Neutralisieren mittels zehntel-normaler Natronlösung. Der Erfolg war:

0,1995 Fettsäuremischung: $6,65 \mathrm{ccm} \mathrm{n} / 10-\mathrm{NaOH}$.

Durchschnittliches Molekulargewicht, gefunden 300.

Berechnet für $\mathrm{C}_{24} \mathrm{H}_{48} \mathrm{O}_{2}, \mathrm{C}_{18} \mathrm{H}_{38} \mathrm{O}_{2}$ und $\mathrm{C}_{18} \mathrm{H}_{32} \mathrm{O}_{2}=303$.

Die Base aus Carnaubon. Mit Säuren oder Alkalien behandelt, liefert Carnaubon eine stickstoffhaltige Base, die wir als Cholin erkannt haben. Diese Base kann nach der Behandlung mit verdünnter Salpetersäure aus der Flüssigkeit durch Phosphorwolframsäure gefällt werden. Die Methode ist aber etwas umständlich und die Ausbeute nicht befriedigend.

Die Methode der Bestimmung, die uns am besten glückte, war die Behandlung mit Baryt. Das Cholin wird durch dieses Reagens zwar teilweise zersetzt, aber man kann sich über den Umfang dieser Zersetzung orientieren, indem man die gebildeten flüchtigen Basen in einer bekannten Menge Säure auffängt. Wir teilen einen solchen Versuch mit: 0,8827 $\mathrm{g}$ Carnaubon, in

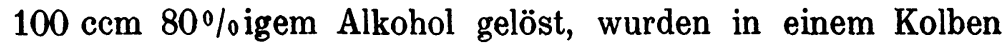
mit $8 \mathrm{~g}$ Baryt auf dem Wasserbad erhitzt. Durch die Mischung wurde ein mittels Kali- und Schwefelsäure gewaschener Luftstrom gesaugt, der zuletzt durch $25 \mathrm{ccm}^{1 / 10-n-S a l z s a ̈ u r e ~ h i n-~}$ durchging. Nach 12 Stunden war der Alkohol verdunstet.

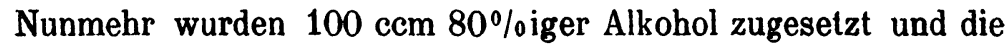
Mischung weitere 12 Stunden lang erhitzt. $1,96 \mathrm{ccm}$ der $\mathrm{n} / 10-$ Säure waren jetzt durch flüchtige Basen neutralisiert, entsprechend 2,69\% Cholin. Wir unterbrachen hierauf den Versuch, gaben zu dem Inhalt des Kolbens $150 \mathrm{ccm}$ Wasser hinzu und leiteten die Mischung Kohlensäure hindurch. Sodann wurde heiß filtriert, der Niederschlag mit heißem Wasser gewaschen und das Filtrat auf etwa $200 \mathrm{ccm}$ eingeengt, mit Salzsäure ganz schwach angesäuert und schließlich bis zur Trockene eingedampft. Der Rückstand wurde mit absolutem Alkohol extrahiert, der Extrakt filtriert und zum Filtrat eine alkoholische Platinchloridlösung hinzugefügt. Es bildete sich sofort ein orangegelber Niederschlag. Am nächsten Tag wurde dieser Niederschlag auf einem Filter gesammelt, mit absolutem Alkohol chlorfrei gewaschen 
und getrocknet. Hierauf wurde er in warmem Wasser aufgelöst und die Lösung in eine gewogene Schale filtriert, eingedampft und bei etwa $95^{\circ}$ bis zum konstanten Gewicht getrocknet. Das Gewicht der so erhaltenen Krystalle betrug 0,2225 g. Auf Cholin berechnet ergibt das 9,93\% des angewandten Carnaubons. Addiert man hierzu das zersetzte Cholin $(2,69 \%)$, so beträgt die Ausbeute $12,62 \%$. Die Theorie verlangt 18,35\%, also annähernd $50 \%$ mehr, als erhalten wurde. Bedenkt man aber, daß das benutzte Carnaubonpräparat 2,93\% Stickstoff statt der theoretischen 3,19\%, somit wahrscheinlich schon Cholin verloren hatte, so wird man nicht $18,35 \%$, sondern nur 16,09\% Cholin erwarten können. Hiernach hätte unsere Bestimmung 78,42\% der zu erwartenden Cholinmenge ergeben. Ein Teil des Carnaubons dürfte überdies der Einwirkung des Baryts entgangen sein wegen der Bildung unlöslicher Barytseifen, die es vor der Zerlegung schützten. Wir glauben, daß dies Resultat zu der Annahme berechtigt, daß sich im Carnaubon zwei Cholingruppen befinden.

Eine Verbrennung des Platindoppelsalzes ergab:

0,2292 Substanz 0,1653 $\mathrm{CO}_{2}, 0,0914 \mathrm{H}_{2} \mathrm{O}, 0,07326 \mathrm{Pt}$.

C $\quad \mathrm{H} \quad \mathrm{Pt}$

Erhalten:

$19,67, \quad 4,45, \quad 31,97$.

Berechnet für $\left(\mathrm{C}_{5} \mathrm{H}_{14} \mathrm{NOCl}_{2} \mathrm{PtCl}_{4}: \quad 19,48, \quad 4,58, \quad 31,65\right.$.

VI. Die Methylgruppen des Carnaubons. In enger Beziehung zu der Menge des Cholins muß die Menge des im Carnaubon enthaltenen Methyls stehen: die Theorie verlangt sechs Methylgruppen. Wir haben eine Methylbestimmung nach der Methode von Hertzig und Meyer versucht. Die Bestimmung ist nicht ganz einwandfrei, denn der Apparat sprang beim Abkühlen nach der dritten Destillation, und wir konnten daher eine vierte Destillation nicht vornehmen. Es ist aber wahrscheinlich, daß die erhaltene Alkyljodidmenge annähernd dem Methylgehalte des Carnaubons entspricht. Die Bestimmung verlief folgendermaßen:

$$
0,7335 \text { Substanz. }
$$

Jodsilber bei der ersten Destillation 0,4177

\begin{tabular}{|c|c|c|c|c|c|}
\hline » & $»$ & » & zweiten & $\triangleright$ & 0,1577 \\
\hline 》 & $\triangleright$ & 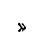 & dritten & » & 0,1159 \\
\hline
\end{tabular}


Diese Zahl entspricht 0,044135 $\mathrm{CH}_{3}$, gleich 6,017\%.

Die theoretische Menge wäre 6,83; das angewandte Carnaubonpräparat enthielt jedoch nur 2,87\% Stickstoff statt 3,19\% und der $\mathrm{zu}$ erwartende Methylgehalt reduziert sich demnach auf $6,144 \%$. Wir dürfen somit annehmen, daß das Carnaubon tatsächlich sechs Methylgruppen enthält.

Der Zucker des Carnaubons. Die Methoden zur Zerlegung des Carnaubons, die wir bis jetzt beschrieben haben sind für die Zuckerbestimmung nicht geeignet; denn der Zucker wird durch die angewandten oxydierenden Mittel stark angegriffen, bezw. vernichtet. Durch Salpetersäure sollte ein Teil des Zuckers in Schleimsäure übergeführt werden, wenn es sich wirklich um Galaktose handelt: in der Tat haben wir kleine Mengen dieser Säure gewonnen. Der Schmelzpunkt der erhaltenen Krystallen war $211-212^{\circ}$, und eine Verbrennung ergab folgende Resultate:

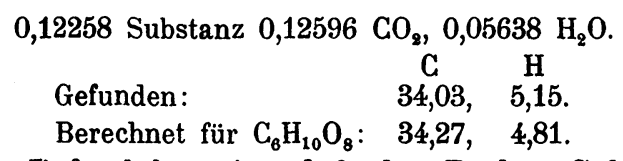

Dieser Befund beweist, daß der Zucker Galaktose oder ein Derivat derselben ist.

Bei der Einwirkung alkalischer Permanganatlösung liefert der Zucker Oxalsäure, doch schwankt die Ausbeute zwischen sehr weiten Grenzen. Kleine Änderungen der Versuchsbedingungen bewirken sehr große Unterschiede in der Menge der erhaltenen Oxalsäure. Einige Resultate, die wir bei solchen Versuchen bekamen, sind aber nicht ohne Interesse und könnten vielleicht eine quantitative Bedeutung haben. Ein solcher Versuch sei deshalb mitgeteilt: 1,5272 g Carnaubon wurden mit $100 \mathrm{ccm}$ Wasser in ein Becherglas gegeben, $50 \mathrm{ccm}$ einer $4 \%$ igen Natriumcarbonatlösung zugegossen und gleich nachher $2 \mathrm{~g}$ Kaliumpermanganat, in $100 \mathrm{ccm}$ Wasser gelöst, unter stetigem Umrühren hinzugefügt. Diese Mischung wurde 70 Stunden lang bei Zimmertemperatur $\left(15-17^{\circ}\right)$ fortdauernd mechanisch gerührt. Der Permanganatüberschuß wurde dann mittels Wasserstoffhyperoxyd zerstört, das Becherglas auf dem Wasserbad erhitzt, das Manganoxyd durch Filtration beseitigt und das 
Filtrat mit Salzsäure angesäuert und erhitzt. Die abgeschiedenen Fettsäuren wurden abfiltriert, das Filtrat mit Natronlauge alkalisch gemacht und dann mit Essigsäure angesäuert. Hierauf wurde die Oxalsäure als Kalksalz gefällt, der Niederschlag auf einen Filter gesammelt und chlorfrei gewaschen und die Menge der Oxalsäure durch Titrieren mit 1/10-n-Chamäleonlösung bestimmt. Die gefundene Oxalsäuremenge betrug 0,10526, entsprechend 6,89\% des angewandten Carnaubons. Wird aus jedem Molekül Zucker ein Molekül Oxalsäure, so würde die Theorie 6,82\% Oxalsäure erfordern.

Sind aber die Bedingungen des Versuches anders gewählt, wird z. B. eine höhere Temperatur oder ein stärkeres Alkali, wie etwa Ätzkali, verwendet, dann ist die Menge der gebildeten Ox்alsäure größer. Unter solchen Bedingungen können jedoch auch die Fettsäuren Oxalsäure liefern. Aus Cholin haben wir keine Oxalsäure durch alkalische Permanganatlösung erhalten. Bei diesem Versuche wurde als Ausgangsmaterial das Platindoppelsalz benutzt.

Die Resultate der Oxalsäurebestimmungen halten wir nur für Fingerzeige, einen sicheren quantitativen Aufschluß geben sie natürlich nicht.

Wie schon bemerkt, ist eine einfache Hydrolyse des Carnaubons durch Säuren schwer zu erzielen; das wäre aber die geeignetste Methode für die Zuckerbestimmung. Wir haben viele Versuche in dieser Richtung angestellt, die Resultate waren indessen niemals quantitative. Ein Versuch ist im folgenden beschrieben :

$5 \mathrm{~g}$ Carnaubon wurden mit 10\% iger Schwefelsäure am Rückflußkühler im Ölbad erhitzt. Die Temperatur des Bades war $118-123^{\circ}$ und die Einwirkung der Säure dauerte 12 Stunden. Die Mischung wurde dann abgekühlt und filtriert und das klare Filtrat mit Baryumcarbonat neutralisiert. Ein abgemessener Teil des Filtrates wurde für die Bereitung des Osazons benutzt, der Rest für die Zuckerbestimmung nach Allihn. Letztere ergab eine Kupfermenge, die 7,52\% des angewandten Carnaubons entsprach. Das deutet auf etwa $4 \%$ Galaktose. Diese Menge kann nur einen Teil des wirklich vorhandenen Zuckers 
darstellen, denn daß die Hydrolyse nicht vollständig war, ist dadurch bewiesen, daß sich keine Fettsäureschichte bildete. Die unlösliche Substanz, die im Hydrolysierungsgemisch schwamm, war ein grauer, schmieriger Schleim statt des zu erwartenden festen Kuchens.

Das Osazon wurde aus Aceton umkrystallisiert; die so erhaltenen, gutausgebildeten Krystalle fingen bei $189^{\circ}$ an sich zu veränderen und waren bei $192^{\circ}$ unter Zersetzung vollständig geschmolzen.

Bis jetzt haben wir wirklich quantitativ verlaufene Zuckerbestimmungen nicht mitzuteilen. Die Spaltungsmethoden, die wir angewandt haben, sind entweder unvollständig gewesen, oder sie vernichteten den Zueker. Ersteren Umstand glauben wir als Stütze für die Annahme betrachten zu dürfen, daß der Zucker als Kern des Carnaubons funktioniert und nicht eine Seitenkette bildet.

Wir haben auch versucht, die Gegenwart einer einfachen Aminogruppe in Carnaubon zu beweisen, indem wir Salpetrigsauredämpfe auf eine Lösung von Carnaubon in Amylalkohol einwirken ließen. Dieser Versuch ist uns nicht gelungen. Das benutzte Carnaubonpräparat enthielt vor dem Versuch 2,93\% Stickstoff. Das Produkt, das sich aus der Lösung bei Zimmertemperatur ausschied, enthielt 3,19\%, also mehr Stickstoff statt weniger. Diese Erfahrung hat uns zuerst darauf aufmerksam gemacht, daß Amylalkohol ein Mittel sein könnte, durch welches man das Carnaubon vielleicht von seinen nächstverwandten Zersetzungsprodukten, die weniger Cholin enthalten, zu trennen vermöchte. Diese Vermutung ist später durch daraufgerichtete Versuche bestätigt worden.

Öfters wiederholte Versuche, Glycerin zu entdecken, sind vergeblich gewesen. Es ist also sieher, daB Glycerin keinen Anteil an der Konstitution des Carnaubons hat.

VIII. Eigenschaften des Carnaubons. Es ist eine weiße, geruch- und geschmacklose, luftbeständige Substanz. Aus alkoholischen oder anderen Lösungen durch KKälte gefällt, macht es den Eindruck, als wäre es krygtallinisch. Unter dem Mikroskop aber kann man keine richtigen Krystalle wahre-

Hoppe-Seyler's Zeitschrift f. physiol. Chemie. LXIV. 
nehmen, wenigstens sieht man nirgends plane Oberflächen, die sich in geraden Linien schneiden. Die Substanz scheint sich vielmehr in Form conchoidaler Scheiben aus den Lösungen auszuscheiden; die mehr oder weniger gekrümmten Falten und Linien, in welchen diese Scheiben zusammentreffen, können Krystallnadeln allerdings sehr ähnlich sehen, allein, daß es wirkliche Krystalle gibt, dafür haben wir bis jetzt keinen Beweis.

Carnaubon ist ganz unlöslich oder äußerst schwer löslich in Äther, auch bei Siedetemperatur; ebenso ist es in Aceton wenig löslich. Methyl-, Äthyl- und Amylalkohol lösen es sehr leicht bei höherer Temperatur, aber schon bei Zimmertemperatur scheidet es sich größtenteils aus, und bei Eisschranktemperatur ist es ganz wenig löslich in diesen Mitteln. Die Löslichkeit in Eisessig, Essigester, Benzol, Toluol, Chloroform und Pyridin ist ähnlich der Löslichkeit in den angegebenen Alkoholen. Eine Mischung von Chloroform und Methylalkohol im Verhältnis $2: 1$ löst bedeutende Mengen des Carnaubons auch bei Zimmertemperatur. Mit Wasser gibt es eine Mischung, die etwas seifenartig und opalescierend ist.

Die Lösung in 50\%igem Alkohol ist neutral gegen Phenolphthalein und die sonstigen üblichen Indikatoren.

Die Lösung in zwei Teilen Chloroform + ein Teil Methylalkohol dreht polarisiertes Licht rechts. Eine Bestimmung ergab: $[\alpha]_{\mathrm{D}}^{25}=+10,2^{0}$.

Auf dem Platinblech erhitzt, schmilzt das Carnaubon zu einer zuerst farblosen Flüssigkeit, die sich bald bräunt, dann mit gelber, rußender, nach verbranntem Fett riechender Flamme brennt und eine schwarze Kohle zurückläßt. Allmählich wird der Rückstand farblos und der zuletzt hinterbleibende Fleck gibt Phosphorsäurereaktion.

Der Schmelzpunkt der Carnaubonpräparate, die wir bei unseren Versuchen meistens benutzten, war $189^{\circ}$ (unkorrigiert).

IX. Zusammenstellung. 1. Aus Rindernieren läßt sich durch Alkohol ein glycerinfreies Triazomonophosphatid extrahieren, das Galaktose oder Aminogalaktose, Carnaubin-, Stearin-, Palmitinund Phosphorsäure und Cholin enthält. Auffallend ist, daß dieses Phosphatid in Äther sehr schwer, fast gar nicht löslich ist: 
2. Die Konstitution dieses Phosphatides scheint lecithinähnlich zu sein, nur bildet Zucker statt Glycerin den Kern der Verbindung, so daß mehr Säuregruppen als im Lecithin gebunden sein können.

3. Wahrscheinlich bildet dieses Phosphatid ein Glied einer Gruppe von ähnlichen Substanzen, die sich von einander durch die Art der Zucker- und der Säuregruppen unterscheiden .

4. Für das besondere Phosphatid, über das wir berichtet haben, wird der Name Carnaubon gewählt.

Anmerkung. Die Arbeit über das Phosphatid, das wir jetzt Carnaubon nennen, wurde zuerst von mir im Jahre 1906 in der Pathologischen Abteilung des University and Bellevue Hospital Medical College, New York, begonnen. Im nächsten Sommer hat Dr. C. J. Robinson, Assistent-Professor der Chemie an demselben Institute, mir freundlichst geholfen, und im Winter 1906-07 hat er allein die Präparierungsmethoden des Carnaubons und einiger anderer Phosphatide weiter ausgebildet. Im Herbst 1907 und seit dem wurde der analytische Teil der Arbeit im Rockefeller Institute for Medical Research weiter geführt. Dort habe ich im Jahre 1908 die Carnaubinsäure isoliert und als solche erkannt. Im Sommer desselben Jahres begann Dr. C. A. Jacobson gemeinsam mit mir über die Substanz zu arbeiten. Ich bin außerdem so vielseitig durch Rat und Tat von Freunden unterstützt worden, daß es unmöglich wäre, jedem meinen gebührenden Dank zu sagen. Ich möchte aber nicht versäumen, Dr. P. A. Levene des Rockefeller Institutes und Dr. H. D. Dakin meine besondere Erkenntlichkeit auszusprechen.

Edward K. Dunham.

Rom, 20. Dezember 1909. 